\title{
A Suspected ectopic pregnancy masking fallopian tube torsion
}

\author{
Jillian J Stack ${ }^{1}$, Suzanne E Davi ${ }^{1}$, Hasthika Ellepola ${ }^{1,2}$, Mano Haran ${ }^{1,2}$ \\ Sri Lanka Journal of Obstetrics and Gynaecology 2012; 34: 163-164
}

\section{Introduction}

Torsion of the fallopian tube is a rare gynaecological entity, affecting only 1 in 1.5 million women ${ }^{1-6,}$ predominantly in the reproductive age group ${ }^{6-7}$. Diagnosis is difficult, owing to the lack of pathognomonic signs, symptoms, laboratory findings and imaging features ${ }^{7}$. The consequent delay in diagnosis and management ${ }^{7}$ is problematic, as time is crucial in order to salvage the tube at laparoscopy, aiming to avoid salpingectomy and thus maximise the fertility of affected women. The present case demonstrates the way in which fallopian tube torsion can mimic other far more common conditions, emphasising the need for clinicians to be hyper-vigilant in the investigation of reproductive-age women with acute lower abdominal pain.

\section{Case report}

A 26 year old female, para 3 (3 normal vaginal deliveries at term), presented with a one day history of heavy per vaginal (PV) bleeding and left iliac fossa (LIF) pain.

The patient's gynaecological history was significant for pelvic inflammatory disease (PID) at 17 years of age, for which she underwent a diagnostic laparoscopy and was treated with antibiotics. All follow up testing had been clear, and the patient was in a long-term monogamous relationship.

The pain was sharp, constant and localised to the LIF, with no associated infective symptoms. She had soaked through four sanitary pads that day, though the bleeding had since settled. On examination, vital signs were stable, and the patient had pain on palpation in the LIF with guarding but no rebound tenderness. A PV exam showed the cervical os to be closed, with old blood in the posterior vaginal fornix. There was left adnexal tenderness but no cervical excitation. Per vaginal discharge appeared physiological.

\footnotetext{
${ }^{1}$ Department of Obstetrics and Gynaecology, Logan Hospital, Queensland, Australia.

${ }^{2}$ Medical School, University of Griffith, Queensland, Australia.

Correspondence: Mohammed Jillian Stack

E-mail: Jillian_Stack@health.qld.gov.au
}

Full blood count and electrolyte and liver function tests were normal. Quantitative BHCG was 1400. A midstream urine sample was clear, and high vaginal and endocervical swab results were pending (though later came back negative). An ultrasound showed a $5 \mathrm{~cm}$ complex lesion within the left adnexa with a haemorrhagic focus, strongly suspicious of a recently ruptured ectopic pregnancy. There was also a trace of free fluid within the left adnexa and Pouch of Douglas. No intrauterine pregnancy was noted, and endometrial appearances were consistent with an organised thrombus rather than products of conception.

The patient proceeded to undergo a laparos-copy for definitive diagnosis and management of a suspected left adnexal ectopic pregnancy. Intraoperatively, the left fallopian tube was distended with what appeared to be an ectopic pregnancy at the distal end, and a left salpingectomy was performed. Additionally, a haemorrhagic cyst was found on the left ovary, for which a cystectomy was performed. The patient had an uncomplicated post-operative recovery and was discharged the following day.

Interestingly, histopathology of the surgical specimens returned several days later, showing oedema and haemorrhage of the fallopian tube wall and lumen, indicative of fallopian tube torsion, with no evidence of an intra-tubal gestation. Follow up of the patient revealed ongoing mild PV bleeding, with a falling BHCG (85 and 36 respectively), suggestive of a concurrent spontaneous miscarriage. At this time, ongoing follow-up was transferred to the care of the patient's general practitioner.

\section{Discussion}

The present case is one that was initially believed to be a straightforward case of a left tubal ectopic pregnancy, but in fact turned out to be the rather more rare condition of a fallopian tube torsion, incidentally associated with a concurrent spontaneous miscarriage.

The pathogenesis of fallopian tube torsion is largely unknown $\mathrm{n}^{6-7,12}$, though predisposing factors have been shown to include intrinsic (tubal) factors such as congenital or acquired pathologies (elongated tube, infection, tumour, ectopic pregnancy) or extrinsic factors such as ovarian or para-ovarian tumours / cysts, pregnancy, trauma or pelvic congestion ${ }^{1,3,6,8-10}$. 
Torsion has been purported to occur sequentially, beginning with blockage of blood and lymphatic supply to the tube due to an aforementioned predisposing factor, leading to congestion, oedema, adnexal enlargement and ultimately torsion of the involved tube $^{3,10}$. Retrospectively, with this information alone, the patient was predisposed to fallopian tube torsion on account of her past history of PID, as well as having a haemorrhagic cyst, though the latter was not apparent until laparoscopy.

Mis-diagnosis is frequent, because symptoms mimic a broad variety of differential diagnoses ${ }^{1-2,9}$, most of which are significantly more common. Symptoms include lower abdominal pain, usually on the side of torsion and radiating to the ipsilateral thigh ${ }^{1,7,9,11}$, and often associated with nausea, vomiting and urinary symptoms ${ }^{1,6-7,11}$. Laboratory findings are often normal, and imaging studies seldom diagnose fallopian tube torsion ${ }^{6,12}$, though ideally sonographic images would reveal a dilated tube with thickened, echogenic walls $^{1,3,6}$ and transvaginal Doppler sonography would show high impedance to flow in the tube ${ }^{1,3,6-7}$. In the present case, diagnosis was even more difficult due to the co-occurring PV bleeding consequent to a spontaneous miscarriage, which along with laboratory and misleading ultrasonographic findings, led to the diagnosis and management of a suspected ectopic pregnancy rather than the true condition at hand.

Laparoscopy remains the gold standard in diagnosis upon suspicion of fallopian tube torsion ${ }^{12}$, and is conveniently also the mainstay of definitive management. De-torsion of the tube is the procedure of choice ${ }^{1-2,6-7}$, with aim to salvage the tube and preserve fertility ${ }^{6-7,10,11}$. Often times, however, the tube is irreversibly ischaemic or gangrenous, prompting the need to perform salpingectomy $y^{1-2,6,10}$. In the present case, the diagnosis was not readily apparent even at laparoscopy, and it was only retrospectively following salpingectomy that fallopian tube torsion was diagnosed.

The lesson learnt from the present case and those others in the literature is that fallopian tube torsion, though a rare event, should always be considered in the differential diagnosis of acute abdominal pain.
Pre-operative diagnosis is difficult and too easily missed due to its non-specific presentation and findings. Importantly, the consequences of delayed diagnosis and management can impede the clinician's ability to preserve tubal function and fertility, highlighting the necessity to increase awareness of this rare gynaecological diagnosis.

\section{References}

1. Bloom RK, Larusso S. Paratubal cyst with isolated torsion of the fallopian tube. J Diagn Med Sonog 2009; 25: 58-61.

2. Raziel A, Mordechai E, Friedler S, et al. Isolated recurrent torsion of the fallopian tube. Hum Reprod 1999; 14: 3000-1.

3. Gross M, Blumstein SL, Chow LC. Isolated fallopian tube torsion: a rare twist on a common theme. Am J Roentgenol 2005; 185: 1590-2.

4. Renjit S, Morale EU, Mathew M. Isolated torsion of a tubal ectopic pregnancy - a rare event. Oman Med J 2008; 23: $289-90$.

5. Shirokane M, Kikuchi F, Satomi M, et al. Isolated torsion of a left normal fallopian tube during pregnancy. J Nipp Med Sch 2011; 78: 40-1.

6. Bayirli R, Oge T, Uysal E, et al. Isolated torsion of the fallopian tube in a 16-year-old adolescent girl. J Obstet Gynecol 2001; 78: 349-61.

7. Krissi H, Shalev J, Bar-Hava I, et al. Fallopian tube torsion: laparoscopic evaluation and treatment of a rare gynecological entity. J Am Board Fam Med 2001; 14: 274-7.

8. Youssef AF, Fayad MM, Shafeek MA. Torsion of the fallopian tube. A clinico-pathological study. Acta Obstet Gynec Scand 1962; 41: 292-309.

9. Duncan RP, Shah MM. Laparoscopic salpingectomy for isolated fallopian torsion in the third trimester. Case Rep Obstet Gynec 2012; 1: 1-3.

10. Lim WH, Roex AJ. Laparoscopic management of a fallopian tubal torsion complicated by a large hydrosalpinx. Int J Wom Heal 2011; 3: 381-4.

11. Vijayaraghavan SB, Senthil S. Isolated torsion of the fallopian tube; the sonographic whirlpool sign. J Ultras Med 2009; 28: 657-62.

12. Mathlouthi N, Jellouli MA, Slimani O, et al. Isolated torsion of the fallopian tube in a woman of reproductive age. J Societe Tunis Sci Medic 2012; 90: 895-6. 